Sir,

\section{Exogenous Cushing's syndrome and topical ocular steroids}

Cushing's syndrome secondary to oral or topical steroids is well known. Cases due to intranasal and inhaled steroids have also been reported. ${ }^{1,2}$ However, Cushing's syndrome as a result of topical ocular steroids is extremely rare. There are only two reported cases in the literature that occurred in infancy and childhood., ${ }^{3,4}$ We present a case of iatrogenic Cushing's syndrome in a young adult with bilateral idiopathic uveitis.

\section{Case report}

An 18-year-old Asian male was referred to the Glaucoma Unit for management of bilaterally raised intraocular pressures (IOP) secondary to uveitis. The patient was otherwise well with no significant past medical history.

His first episode of uveitis and elevated IOP occurred at age 12 years (IOP $41 \mathrm{mmHg}$ LE - settled with a short course of topical steroids). He was then trouble free until 16 years of age when he was noted to have elevated left IOP of $43 \mathrm{mmHg}$ and bilateral chronic anterior uveitis. The patient weighed $67.3 \mathrm{~kg}$ at this stage and the uveitis was maintained using twice daily prednisolone acetate $1 \%$ to both eyes until presentation 10 months later.

On presentation to the Glaucoma Unit, best-corrected visual acuity was 6/9 RE and 6/12 LE. The patient had bilateral high myopia with $-8.00 \mathrm{D}$ RE and -11.00D LE. Intraocular pressures were raised at $41 \mathrm{mmHg} \mathrm{RE}$ and $42 \mathrm{mmHg}$ LE. There was a left-sided relative afferent pupillary defect. Slit lamp examination revealed $1+$ cells and $1+$ flare in both eyes as well as numerous old keratic precipitates. There were no cells in the vitreous of either eye. Posterior subcapsular lens opacities were present bilaterally. Fundal examination showed a cup : disc ratio of $0.4 \mathrm{RE}$, and end-stage glaucomatous cupping of 0.95 LE. He was treated with maximally tolerated medical glaucoma therapy including oral acetazolamide and a decreasing dose of hourly $1 \%$ prednisolone acetate.

In the next 6 months, he underwent a left augmented trabeculectomy with mitomycin-c and sequential phacoemulsification with IOL implantation. During this period, he received a total of $2.5 \mathrm{~g}$ of intravenous pulsed methylprednisolone preoperatively and varying doses of topical ocular steroids (Figures 1-3).

During a visit 8 months after presentation, the treating consultant noted the patient's weight gain with the development of axillary and abdominal striae. The patient attributed the weight gain to reduced physical activities. He denied any systemic or recreational steroid use.
On examination, the patient was afebrile with a heart rate of 60 beats $/ \mathrm{min}$. Blood pressure was $123 / 57 \mathrm{mmHg}$ and the random blood glucose was $4.4 \mathrm{mmol} / 1$. His weight at this stage had increased to $91 \mathrm{~kg}$. Physical signs consistent with Cushing's syndrome included a moonlike face, truncal obesity, and numerous body striae (Figures 4-6). There was no proximal muscle weakness. Cranial nerve examination was normal and no mass was

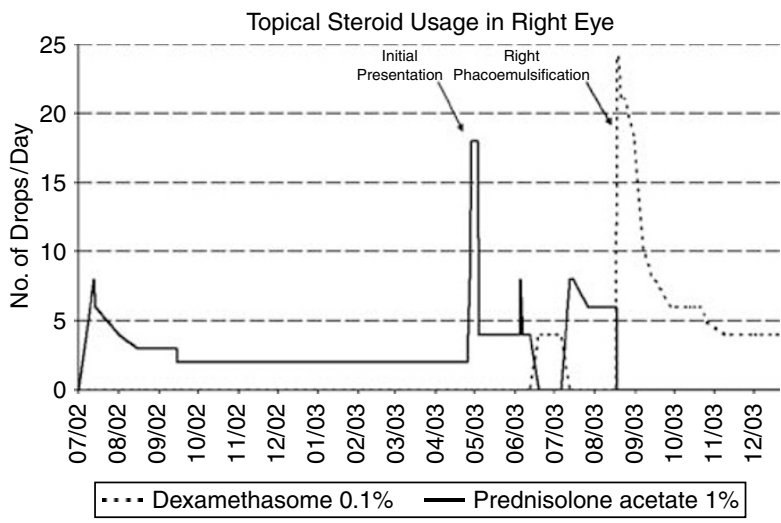

Figure 1 Topical steroid usage in the right eye.

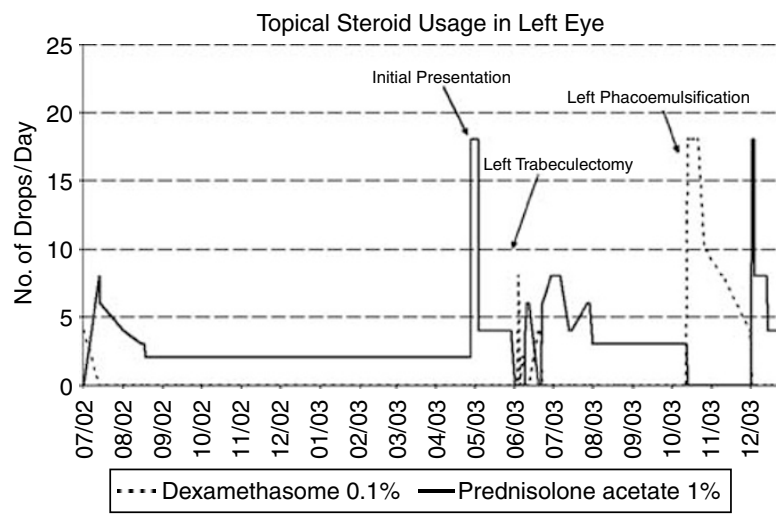

Figure 2 Topical steroid usage in the left eye.

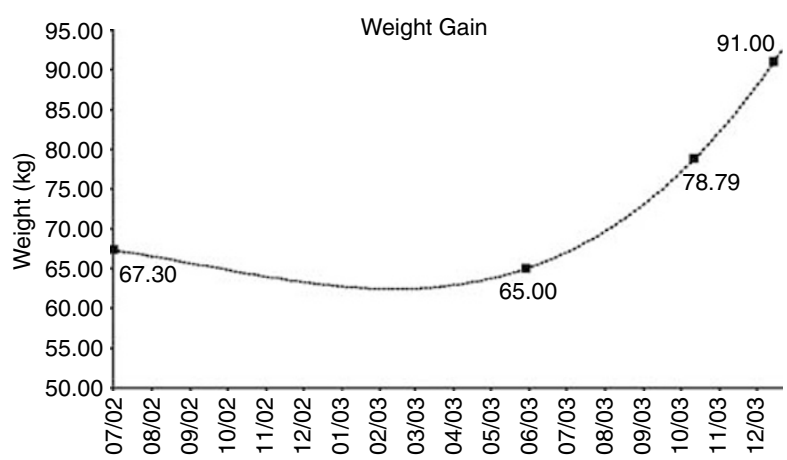

Figure 3 Weight gain during topical steroid use. 
felt in the abdomen. Three consecutive 24-h urinary free cortisol levels were low at $75,<42$, and $79 \mathrm{nmol} /$ day. Early morning blood cortisol was also low at $37 \mathrm{nmol} / \mathrm{l}$. The patient had normal thyroid function and normal fasting blood glucose. Other routine haematological and biochemical tests revealed no abnormalities. He was referred to the endocrinology unit for further opinions. A subsequent synacthen stimulation test failed to raise the cortisol levels significantly. Cortisol levels before

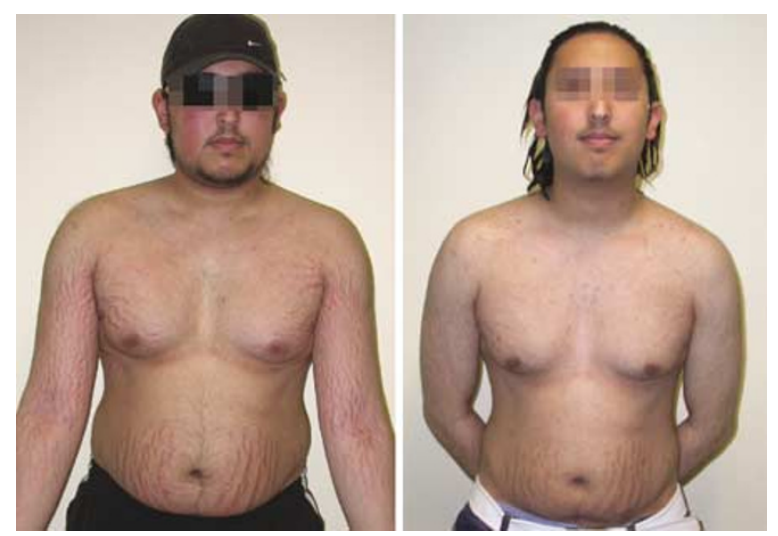

Figure 4 Picture of patient at diagnosis and at time of ceasing topical ocular steroids. Features of Cushing's syndrome became less evident.
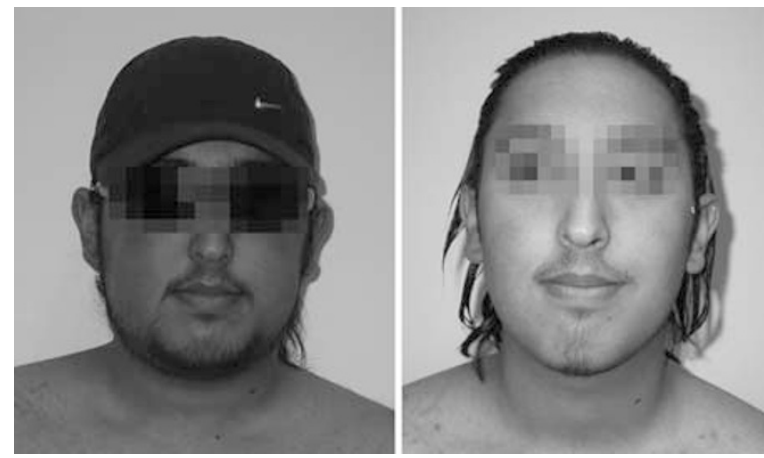

Figure 5 Moon-like face improved after ceasing topical ocular steroids.
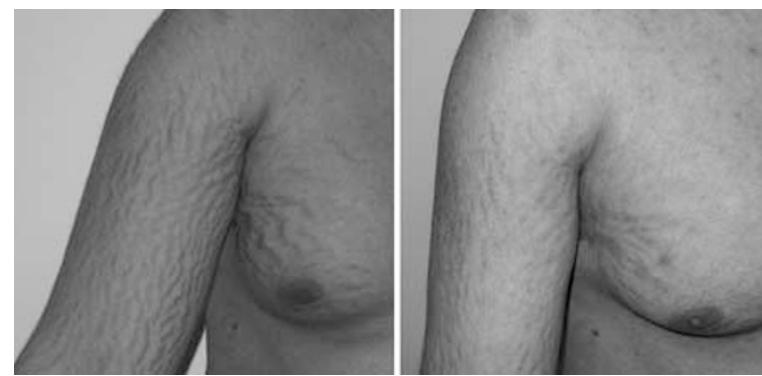

Figure 6 Axillary striae became less pronounced after withdrawal of topical ocular steroids. synacthen and at 30 and 60 min after synacthen administration were $<28,65$, and $69 \mathrm{nmol} / 1$, respectively. A diagnosis of exogenous Cushing's syndrome possibly secondary to topical ocular steroids was made. Imaging of the pituitary or the adrenal glands was not performed in view of the biochemical findings and the close temporal relationship between weight gain and topical ocular steroid use.

The patient was managed in close collaboration with the endocrine and rheumatology units. Methotrexate was commenced to allow gradual reduction of topical ocular steroids while maintaining control of his severe refractory uveitis. At the same time, synacthen stimulation test was repeated on several occasions to assess the response of the hypothalamo-pituitary-adrenal axis. Oral supplemental hydrocortisone was given when required to prevent Addisonian crisis. At 12 months after the diagnosis of exogenous Cushing's syndrome, the patient has been weaned off all topical ocular steroids and his weight has decreased to $76.5 \mathrm{~kg}$. Also, physical features of Cushing's syndrome has become less evident (Figures 4-6). Synacthen stimulation tests performed during this period revealed a slow recovery of the hypothalamo-pituitary-adrenal axis (Figure 7). Cortisol levels from the synacthen stimulation tests were 243, 310, and $383 \mathrm{nmol} / \mathrm{l}$ after 5 months and 296, 407, and $434 \mathrm{nmol} / 1$ after 7 months. Further synacthen stimulation tests will be performed when the patient has been off all steroids for a considerable period of time.

\section{Comment}

Iatrogenic Cushing's syndrome secondary to topical ocular steroids has never been described in an adult. Previous cases in the literature involved an infant and an

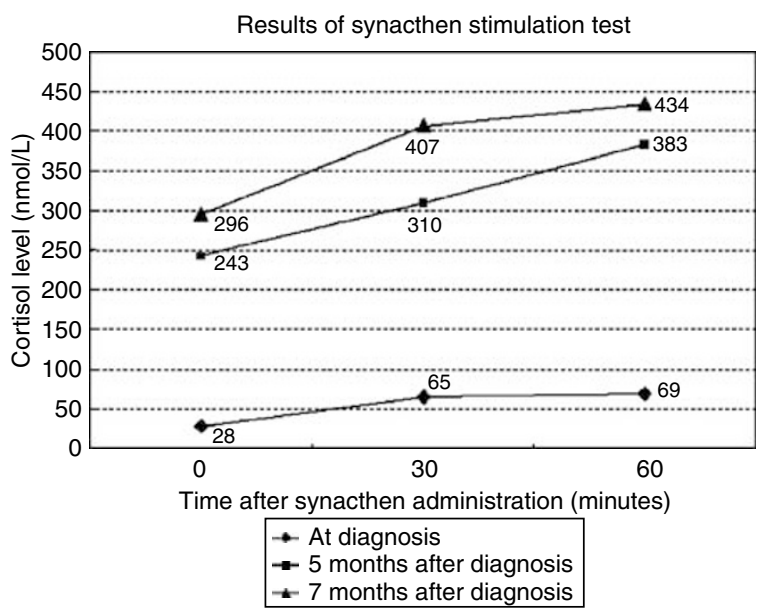

Figure 7 Results of synacthen stimulation tests during gradual reduction of topical ocular steroids. 
11-year-old boy. It is unclear why these patients developed Cushing's syndrome when there are many others using similar amounts of topical ocular steroids. It has been suggested that in patients with Cushing's syndrome, the pituitary gland responds differently from others who are given the same dose of steroid. ${ }^{5}$ In this reported case, the diagnosis of iatrogenic Cushing's syndrome with a depressed hypothalamo-pituitaryadrenal axis was confirmed by a low basal cortisol level and the inability to raise cortisol production after synacthen. The patient's weight gain also correlated well to the increased topical steroids usage after initial presentation (Figure 3). The use of preoperative intravenous methylprednisolone and bursts of higher topical steroid usage may have contributed to the development of Cushing's syndrome. Interestingly, Cushing's syndrome has been reported in a 24 -year-old male after $60 \mathrm{mg}$ of epidural methylprednisolone. ${ }^{6}$

When there is a suspicion of Cushing's syndrome, it is essential to warn the patient regarding possible lifethreatening infections and the need to seek immediate medical help if unwell. Also, the patient needs to be advised on the correct drop instillation technique including punctal occlusion to reduce systemic steroid absorption. The Glaucoma Unit has started to obtain baseline weight, blood pressure, and glucose routinely in patients likely to be on long-term topical steroid therapy. The management of this patient involved close liaison with the endocrinology and rheumatology units and careful monitoring of the hypothalamo-pituitary-adrenal axis when withdrawing steroids. This would not have been possible without the use of nonsteroidal-based systemic immunosuppression to control the patient's uveitis. The patient was commenced on systemic immunosuppression with methotrexate in close collaboration with the endocrine and rheumatology units. The aim of this was to allow a gradual reduction in the topical ocular steroids while maintaining adequate control of his severe refractory chronic anterior uveitis.

This case of Cushing's syndrome illustrates the potential for unusual and serious extraocular side effects of topical ocular steroids. It is essential that the treating ophthalmologist be aware of this to avoid life-threatening complications.

\section{Acknowledgements}

We have no financial or propriety interest in any material or method mentioned.

\section{References}

1 Wilson AM, Blumsohn A, Jung RT, Lipworth BJ. Asthma and Cushing's syndrome. Chest 2000; 117: 593-594.
2 Homer JJ, Gazis TG. Cushing's syndrome induced by betamethasone nose drops. In rhinological disease betamethasone should be regarded as systemic corticosteroid. BMJ 1999; 318: 1355.

3 Ozerdem U, Levi L, Cheng L, Song MK, Scher C, Freeman WR. Systemic toxicity of topical and periocular corticosteroid therapy in an 11-year-old male with posterior uveitis. Am J Ophthalmol 2000; 130: 240-241.

4 Romano PE, Traisman HS, Green OS. Fluorinated corticosteroid toxicity in infants. Am J Ophthalmol 1977; 84: 249-250.

5 Frey FJ, Amend Jr WJ, Lozada F, Frey BM, Benet LZ. Endogenous hydrocortisone, a possible factor contributing to the genesis of cushingoid habitus in patients on prednisone. J Clin Endocrinol Metab 1981; 53: 1076-1080.

6 Tuel SM, Meythaler JM, Cross LL. Cushing's syndrome from epidural methylprednisolone. Pain 1990; 40: 81-84.

MY-M Chiang1', M Sarkar², JM Koppens'1, J Milles²

and P Shah

${ }^{1}$ Birmingham and Midland Eye Centre, Birmingham, UK

${ }^{2}$ Good Hope Hospital, Birmingham, UK

Correspondence: P Shah, Birmingham and

Midland Eye Centre, City Hospital NHS Trust,

Dudley Road, Birmingham B18 7QH, UK

Tel: + 441215076808 ;

Fax: + 441215076791

E-mail: p.f.shah@talk21.com

Eye (2006) 20, 725-727. doi:10.1038/sj.eye.6701956; published online 18 November 2005

Sir,

Reply to intrevitreal triamcinolone for branch retinal vein occlusion

I read the article by Jonas et $a l^{1}$ on 'Branch retinal vein occlusion treated by intravitreal triamcinolone acetonide'. I disagree with their conclusion that an intravitreal injection of triamcinolone can increase visual acuity in patients with branch retinal occlusion. If one looks in Tables 2 and 3 at the preoperative and study end visual acuities of the study and control groups there is no significant change in acuity over the study period for either group. In addition, there is no significant difference between the groups at either time point. Given that a total of 19 comparisons of mean visual acuity were performed I suggest that a correction of the level of significance should have been performed. ${ }^{2}$ This would have meant that none of the comparisons of acuity at intermediate time points in Tables 2 and 3 would have reached statistical significance. 